\title{
Dynamic Equilibria in Statistical and Nonlinear Physics of Uniform Stress Rotating Space Elevators (USRSE)
}

\author{
Leonardo Golubović ${ }^{1} \&$ Steven Knudsen ${ }^{1}$ \\ ${ }^{1}$ Physics and Astronomy Department, West Virginia University, Morgantown, USA \\ Correspondence: Leonardo Golubović, Physics and Astronomy Department, West Virginia University, POB 6315, \\ Morgantown, WV 26506, USA. E-mail: lgolubov@wvu.edu
}

Received: November 7, 2019

Accepted: November 25, 2019 Online Published: December 17, 2019

doi:10.5539/apr.v11n6p56

URL: http://dx.doi.org/10.5539/apr.v11n6p56

\begin{abstract}
The discovery of ultra-strong materials such as carbon nanotubes and diamond nano-thread structures has recently motivated an enhanced interest for the physics of Space Elevators connecting the Earth with outer space. A new concept has recently emerged in space elevator physics: Rotating Space Elevators (RSE) [Golubović, L. \& Knudsen, S. (2009). Classical and statistical mechanics of celestial scale spinning strings: Rotating space elevators. Europhysics Letters 86(3), 34001.]. Objects sliding along rotating RSE string (sliding climbers) do not require internal engines or propulsion to be transported from the Earth's surface into outer space. Here we address the physics of a special RSE family, Uniform Stress Rotating Space Elevators (USRSE), characterized by constant tensile stress along the string. From the point of view of materials science, this condition provides the best control of string's global integrity. We introduce an advanced analytic approach to obtain the dynamic equilibrium configurations of USRSE strings. We use our results to discuss the applications of USRSE for spacecraft launching.
\end{abstract}

Keywords: Space Elevators, Nonlinear Dynamics, Statistical Mechanics, Space Travel, Asymptotic Methods

\section{Introduction}

Space Elevators connecting the Earth with outer space have gained an increased attention in this millennium (Edwards \& Westling, 2003). This interest emerged in part due to the discovery of ultra-strong materials such as carbon nanotubes (Yu et al., 2000a, b) and diamond nano-thread structures (Fitzgibbons et al., 2014), that can be used to build a space elevator. On the physics side, space elevators are also interesting because they are cosmic scale realizations of physical systems with reduced dimensionality such as the strings, polymers, and membranes (Kardar, 2007; Nelson, 2002; Nelson, Piran \& Weinberg, 1988).

Recently, a new concept has emerged in the area of applied non-linear and statistical physics: Rotating Space Elevator (RSE) (Golubović \& Knudsen, 2009; Knudsen \& Golubović, 2014, 2015, 2016). The RSE is a double rotating floppy string reaching outer space locations. Interestingly, objects sliding along the RSE rotating string loops (sliding climbers) do not require internal engines or propulsion to be transported far away from the Earth's surface; see Figure 1(a). The RSE thus solves a major problem in space elevator physics which is how to supply energy to the climbers moving along space elevator strings. In this study we address the physics of a special RSE family, Uniform Stress Rotating Space Elevators (USRSE), introduced by Golubović and Knudsen (2009). USRSE is characterized by constant tensile stress along the string. From the point of view of materials science, this condition provides the best control of string's global integrity over its entire length. In this work, we introduce an advanced analytic approach to obtain dynamic equilibrium configurations of USRSE strings. We then use our results to discuss the applications of USRSE for spacecraft launching.

\section{USRSE Dynamic Equilibria}

The RSE concept is based on specially chosen ("magical") line mass density of the floppy string such that the rotating string is in dynamic equilibrium (in the non-inertial frame rotating with the planet) in the presence of planetary gravitational forces and inertial forces involving the internal centrifugal force due to string rotation and the centrifugal force due to the planet's rotation (Golubović \& Knudsen, 2009; Knudsen \& Golubović, 2014, 2015). These rotations and the planet's gravity cause a tension in the string. USRSE is a special family of RSEs such that the local tensile stress (tension per unit area of the string cross section) is uniform, i.e., constant along the string. In the context of traditional space elevators (Pearson, 1975), the constant stress condition provides a simple and 
very good control of the string's global integrity, in view of a finite tensile strength (breakup tensile stress) of realistic string materials such as carbon nanotubes (Yu et al., 2000a, b).

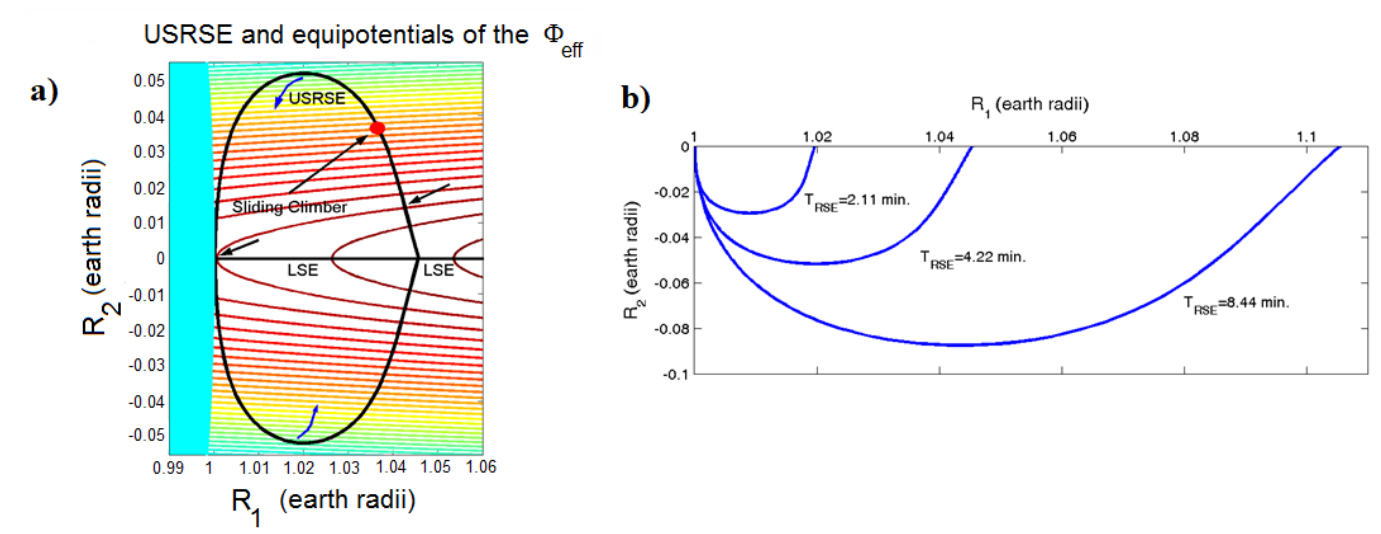

Figure 1. In panel (a), we display the USRSE (attached to a linear space elevator, LSE, along $\mathrm{R}_{1}$ axis) with $T_{R S E}=$ 4.22 min time period of rotation around $\mathrm{R}_{1}$ axis. The system is attached to the Earth or another planet (blue) at its equator. The figure also includes the equipotentials of the effective potential in Eq. (2). Sliding climbers oscillate

between two turning points (indicated by straight arrows) that are on the same equipotential (adapted from

Golubović \& Knudsen (2009)). In panel (b), we display several USRSE forms numerically found at different

time periods $T_{R S E}$, all obtained at the stress parameter $\widetilde{K}=\frac{1}{4}$ (adapted from Knudsen \& Golubović, 2014)

The USRSE condition of uniform stress imposes a constraint on the string form in its dynamic equilibrium, determined by solving the differential equation (Golubović \& Knudsen, 2009; Knudsen \& Golubović, 2014),

$$
\frac{d^{2} R_{2}}{d R_{1}^{2}}=-\frac{1}{K}\left[1+\left(\frac{d R_{2}}{d R_{1}}\right)^{2}\right]\left[a_{2}\left(R_{1}, R_{2}\right)-a_{1}\left(R_{1}, R_{2}\right) \frac{d R_{2}}{d R_{1}}\right]
$$

The function $R_{2}\left(R_{1}\right)$ describes the string's dynamic equilibrium form (as in the snapshot in Figure 1). In Eq. (1), the stress parameter $K=p / \rho$, where $p$ is the uniform tensile stress in the string and $\rho$ is the mass density of the string's material. Noteworthy is that $K$ has dimensions of velocity squared. In Eq. (1), $a_{i}=-\partial \Phi_{e f f} / \partial R_{i}$ $(i=1,2)$, where $\Phi_{e f f}$ is the effective potential,

$$
\Phi_{e f f}=-\frac{g R_{E}^{2}}{\sqrt{R_{1}^{2}+R_{2}^{2}}}-\frac{1}{2} \Omega_{E}^{2} R_{1}^{2}-\frac{1}{2}\left(\Omega_{R S E}^{2}+\frac{\Omega_{E}^{2}}{2}\right) R_{2}^{2}
$$

Here, $\Omega_{R S E}$ is the USRSE angular velocity of its internal rotation about $\mathrm{R}_{1}$ axis (see Figure 1(a)), $\Omega_{E}$ is the angular velocity of the planet, $\mathrm{R}_{E}$ is the planet's radius and $g$ is the gravitational acceleration on its surface. We note that, in this context, the string tensile stress is best characterized by dimensionless stress parameter $\widetilde{K}=$ $K / v_{1}^{2}$ where $v_{1}=\sqrt{g R_{E}}$ is the planet's $1^{\text {st }}$ cosmic speed. Clearly from Eqs. (1) and (2), finding the USRSE dynamic equilibrium form is a complex problem full of secrets and mysteries. The problem was addressed numerically in the past, for the purposes of numerical simulations of USRSE and sliding climbers dynamics (Golubovic \& Knudsen, 2009; Knudsen \& Golubović, 2014). Figure 1 displays some numerically obtained forms of USRSE. Thus, Figure 1(a) shows numerically found USRSE dynamic equilibrium shape for $T_{R S E}=\frac{2 \pi}{\Omega_{R S E}}=$ $4.22 \mathrm{~min}$ and $\widetilde{K}=1 / 4$ corresponding to the USRSE string tensile stress $p=\widetilde{K} \varrho v_{1}^{2}=20.24 G P a$ (for the Earth's $1^{\text {st }}$ cosmic speed, $v_{1} \approx 7.9 \mathrm{~km} / \mathrm{sec}$ ) if the USRSE is made of carbon nano-tubes (CNT) with $\rho=$ $1,300 \mathrm{~kg} / \mathrm{m}^{3}$. Thus, importantly, the tensile stress $p=20.24 \mathrm{GPa}$ of this USRSE is significantly smaller than the tensile strengths (breakup stresses) $p_{\max } \approx 60 \mathrm{GPa}$ of single-wall CNT, and $p_{\max } \approx 150 \mathrm{GPa}$ of multi- 
wall CNT (Yu et al., 2000a, Yu et al., 2000b). So, the USRSE in Fig 1(a) is technologically achievable with modern day materials. Moreover, interestingly, the USRSE in Fig 1(a) (with $\widetilde{K}=\frac{1}{4}$ and $T_{R S E}=4.22 \mathrm{~min}$ ) is capable to launch spacecraft into the Earth's orbit (Golubović \& Knudsen, 2009; Knudsen \& Golubović, 2014). It is however certainly safer to use an USRSE with even smaller stress parameter, $\widetilde{K}<1 / 4$. Here, we will address the question about the feasibility of such smaller stress USRSEs to launch spacecraft.

In this study we analytically address the problem of finding the dynamic equilibrium forms of rotating USRSE strings. Having in mind the possibility of dealing with USRSE systems not just on the Earth but also on other planets (or asteroids) poses the shape problem in the five-dimensional parameter space of the free parameters $\left(\widetilde{K}, \Omega_{R S E}, \mathrm{R}_{E}, g, \Omega_{E}\right)$ which determine USRSE shape and size by solving Eq. (1). Many questions can be asked about the solutions to Eq. (1). For example, one would like to have a general analytic result for the maximum value of the rotating string speed, $v_{\max }$, which occurs at the string point with the maximum value of the $R_{2}$ coordinate in Figure 1 (called as apex in the following). This point on the string plays the major role in the potential USRSE applications for launching spacecraft (Knudsen \& Golubović, 2014), since the maximum rotating spring speed $v_{\max }=\Omega_{R S E} \quad R_{2, \max }$ is reached only at the apex.

Numerical results displayed in Figure 1(b) indicate that USRSE linear sizes, such as $R_{2, \text { max }}$, decrease relative to the planet radius $\mathrm{R}_{E}$, with decreasing USRSE time period $T_{R S E}=\frac{2 \pi}{\Omega_{R S E}}$. This feature suggests approaching the shape problem in Eqs. (1) and (2) by expanding the effective potential in Eq. (2) in powers of $\frac{x}{R_{E}}=\frac{R_{1}-R_{E}}{R_{E}}$ and $\frac{y}{R_{E}}=\frac{R_{2}}{R_{E}}$. To the leading order in this systematic expansion we find,

$$
\Phi_{e f f}(x, y)=\Phi_{e f f}(0,0)+g^{\prime} x-\frac{1}{2}\left(\Omega_{R S E}^{\prime}\right)^{2} y^{2}
$$

while Eq. (2) assumes the form,

$$
\frac{d^{2} y}{d x^{2}}=-\frac{1}{K}\left[1+\left(\frac{d y}{d x}\right)^{2}\right]\left[\left(\Omega_{R S E}^{\prime}\right)^{2} y+g^{\prime} \frac{d y}{d x}\right]
$$

In Eqs (3) and (4),

$$
g^{\prime}=g\left(1-\Delta_{1}\right),\left(\Omega_{R S E}^{\prime}\right)^{2}=\Omega_{R S E}^{2}-\Omega_{1}^{2}\left(1-\frac{\Delta_{1}}{2}\right)
$$

where $\Delta_{1}=\left(\frac{\Omega_{E}}{\Omega_{1}}\right)^{2}=\left(\frac{T_{1}}{T_{d a y}}\right)^{2}$, with $T_{\text {day }}=\frac{2 \pi}{\Omega_{E}}$ (sidereal day), and $T_{1}=\frac{2 \pi}{\Omega_{1}}=\frac{2 \pi R_{E}}{v_{1}}$ (the period of the circular orbit with the radius $\left.R_{E}\right)$. For the Earth, $T_{d a y}=1436.07 \mathrm{~min}$ and $T_{1}=84.44 \mathrm{~min}$; thus $\Delta_{1}=(0.0588)^{2}=$ 0.00346 . Such typically small $\Delta_{1}$ values characterize all planets, to assure they do not fall apart due to their own rotation. We stress that the analysis in this paper is general and it can be applied to any planet. We also note that $\Omega_{R S E}^{\prime} \cong \Omega_{R S E} \gg \Omega_{1}$, i.e., $\mathrm{T}_{R S E}^{\prime} \cong \mathrm{T}_{R S E} \ll \mathrm{T}_{1}$; see Figure 1(b). The necessity of this condition will be elucidated in this study. We proceed by rescaling the $(x, y)$ coordinates in Eq. (4),

$$
x=\lambda X, y=\lambda Y
$$

with the length scale

$$
\lambda=\frac{\sqrt{K}}{\Omega_{R S E}^{\prime}}=\frac{v_{1} \sqrt{\widetilde{K}}}{\Omega_{R S E}^{\prime}}
$$

This rescaling transforms Eq. (4) into the dimensionless form, 


$$
\frac{d^{2} Y}{d Y^{2}}=-\left[1+\left(\frac{d Y}{d X}\right)^{2}\right]\left[Y+\sqrt{\alpha} \frac{d Y}{d X}\right]
$$

having a single dimensionless parameter, $\alpha$, defined by,

$$
\alpha=\frac{\left(g^{\prime}\right)^{2}}{K\left(\Omega_{R S E}^{\prime}\right)^{2}}=\frac{\left(g^{\prime}\right)^{2}}{v_{1}^{2} \widetilde{K}\left(\Omega_{R S E}^{\prime}\right)^{2}}
$$

$\alpha$ parameter will play the central role in all of the following discussions. Using the above definitions, this parameter can also be expressed by the relation,

$$
\alpha=\frac{1}{\widetilde{K}} \frac{\left(\frac{T_{R S E}}{T_{1}}\right)^{2}}{1-\left(\frac{T_{R S E}}{T_{1}}\right)^{2}\left(1+\Delta_{2}\right)}
$$

which can be inverted to express the RSE period in terms of $\alpha$,

$$
\frac{T_{R S E}}{T_{1}}=\frac{\sqrt{\alpha \widetilde{K}}}{\sqrt{1+\alpha \widetilde{K}\left(1+\Delta_{2}\right)}}
$$

where, $\Delta_{2}=\frac{3}{2} \Delta_{1}\left(1-\frac{2}{3} \Delta_{1}\right) /\left(1-\Delta_{1}\right)^{2}$. With the previously mentioned $\Delta_{1}=0.00346$ for the Earth, one finds $\Delta_{2}=0.00520$.

Apparently from Eq. (10), small $\alpha$ is reached if $T_{R S E} \ll T_{1}$. We note this because it will be revealed in this study that applying USRSE for spacecraft launching is only feasible with USRSEs having very small $\alpha$ values $\left(\alpha \sim 10^{-2}\right.$ or even smaller). Here, we have in mind realistic string materials (such as carbon nanotubes, Yu et al., 2000a, Yu et al., 2000b) with limited permissible values of the stress parameter $\widetilde{K}$ (such as the aforementioned $\widetilde{K}=\frac{1}{4}$ or even smaller).

Interestingly, by the above definitions, $\alpha$ can also be expressed as,

$$
\alpha=\frac{1}{1+\left[\left(\frac{T_{\max }}{T_{R S E}}\right)^{2}-1\right]\left(1+\widetilde{K}\left(1+\Delta_{2}\right)\right)}
$$

with,

$$
T_{\max }=\frac{T_{1}}{\left(1-\Delta_{1}\right) \sqrt{\frac{1}{\widetilde{K}}+1+\Delta_{2}}}
$$

The timescale $T_{\max }$ in Eq. (13) plays a special role in the RSE physics. A sliding climber starting at rest at the Earth (or another planet) surface will accomplish its travel to outer space (as depicted in Fig 1(a)) only if $T_{R S E}<$ $T_{\max }$ (Knudsen \& Golubović, 2014). Conversely, if $T_{R S E}>T_{\max }$, an energy barrier due to gravity will force the climber to remain localized at the planet surface (Knudsen \& Golubović, 2014). By Eq. (12), we can see that the climbing threshold $\left(T_{R S E}=T_{\max }\right)$ exactly corresponds to $\alpha=1$. Therefore, USRSE can function as a space elevator only if $\alpha<1$. However, as will be shown in this study, such an USRSE can be used for spacecraft launching only under a stronger condition that $\alpha$ is smaller than a characteristic $\alpha$ value that turns out to be much smaller than 1 for permissible values of the dimensionless stress parameter $\widetilde{K}$ (which are dictated by the tensile strength of the material used to make USRSE string); see Sec. 4 and Figures 4(a) and 4(b) therein.

By the above definitions, the characteristic USRSE length scale $\lambda$ in Eq. (7) satisfies the relation,

$$
\frac{\lambda}{R_{E}}=\frac{\widetilde{K}}{1-\Delta_{1}} \sqrt{\alpha}
$$

Thus, the apex $R_{2}$ coordinate, $R_{2, \max }=\lambda Y_{\max }$ is found to satisfy the relation

$$
\frac{R_{2, \max }}{R_{E}}=\frac{\widetilde{K}}{1-\Delta_{1}} \sqrt{\alpha} Y_{\max }(\alpha)
$$

where $Y_{\max }(\alpha)$ is the maximum value of the USRSE shape function $Y(X)$ obtained by solving Eq. (8) for a given $\alpha$ value. By the above definitions one also finds that maximum rotating string speed (reached at the apex), 
$v_{\max }=\Omega_{R S E} R_{2, \max }$ satisfies the relation,

$$
\frac{v_{\max }}{v_{1}}=\frac{\sqrt{1+\alpha \widetilde{K}\left(1+\Delta_{2}\right)}}{1-\Delta_{1}} \sqrt{\widetilde{K}} Y_{\max }(\alpha) .
$$

It is very easy to see that the two important ratios in Eqs. (15) and (16) are simply related to each other,

$$
\frac{R_{2, \max }}{R_{E}}=\frac{T_{R S E}}{\mathrm{~T}_{1}} \frac{v_{\max }}{v_{1}}
$$

In the next section, we will obtain an analytic expression for the function $Y_{\max }(\alpha)$. We find that this function actually diverges in the small- $\alpha$ limit; see Figure 2 in the following. This result, in connection with Eq. (16), is fundamental since it shows that $v_{\max }$ will generally exceed the $1^{\text {st }}$ cosmic speed $v_{1}$, for any $\widetilde{K}$ value, provided $\alpha$ is small enough! Thus, USRSE system can be used for spacecraft launching provided $\alpha$ is small enough. We will also find that the value of $\sqrt{\alpha} Y_{\max }(\alpha)$ approaches zero in the small- $\alpha$ limit; see Figure 2 in the following. This result, in connection with Eq. (15), is significant since it shows that USRSE linear sizes shrink relative to the planet radius $R_{E}$. This self-consistently justifies the expansion yielding the Eqs. (3), (4), end, eventually, Eq. (8) which is central to this study. We also note that, to the leading order in small- $\alpha$ limit, Eqs. (10), (11) and (16) reduce to simple forms,

$$
\alpha \cong \frac{1}{\widetilde{K}}\left(\frac{T_{R S E}}{T_{1}}\right)^{2}
$$

i.e., $T_{R S E} / T_{1} \cong \sqrt{\alpha \widetilde{K}}$, and,

$$
\frac{v_{\max }}{v_{1}} \cong \frac{1}{1-\Delta_{1}} \sqrt{\widetilde{K}} Y_{\max }(\alpha)
$$

\section{Asymptotic Analysis of USRSE in Small-a Limit}

The USRSE shape differential equation Eq. (8) is strongly nonlinear at large string tangent slopes relative to the $\mathrm{X}$-axis, $m=\frac{d Y}{d X}=\tan (\theta) \gg 1$. These large or even infinite slopes $\left(\theta=90^{\circ}\right)$ actually play the major role for USRSE string morphology as discussed in the following. See Figure 1; note that the string is tangential to the planet; therefore $\theta=90^{\circ}$ at the USRSE string tying point to the planet. To initiate our analysis, we rewrite the USRSE shape equation Eq. (8) in this form,

$$
\frac{\frac{d}{d X}\left(\frac{d Y}{d X}\right)}{1+\left(\frac{d Y}{d X}\right)^{2}}+Y=-\sqrt{\alpha} \frac{d Y}{d X}
$$

By multiplying Eq. (20) by $d Y / d X$, the resulting LHS turns out to have the form of a total derivative,

$$
\frac{d}{d X}\left[\frac{1}{2} \ln \left(1+\left(\frac{d Y}{d X}\right)^{2}\right)+\frac{Y^{2}}{2}\right]=-\sqrt{\alpha}\left(\frac{d Y}{d X}\right)^{2}
$$

Note that for $\alpha=0$, the quantity in the square brackets on the LHS (named in the following as $E$, quasi-energy),

$$
E=\frac{1}{2} \ln \left(1+\left(\frac{d Y}{d X}\right)^{2}\right)+\frac{Y^{2}}{2}
$$

is constant (conserved) and the Eq. (21) becomes integrable. This feature motivates the asymptotic analysis USRSE morphology in small- $\alpha$ limit done in the following. Prior to this, it is illuminating to note exact analogy of our physical problem in Eqs. (20) and (21), to the dissipative particle dynamics (in quasi-time X, with the quasi-particle position $\mathrm{Y}(\mathrm{X})$ and quasi-velocity $m=\frac{d Y}{d X}$ ) of a quasi-particle characterized by an anharmonic form of kinetic energy, $\frac{1}{2} \ln \left(1+\left(\frac{d Y}{d X}\right)^{2}\right)$, moving in the harmonic potential $\frac{Y^{2}}{2}$. In this analogy, the RHS of Eq. (21) is the 
dissipative power (loss rate of the quasi-energy $E$ ) due to quasi-viscous friction force $-\sqrt{\alpha} \frac{d Y}{d X}$. Thus, the problem of the USRSE shape is closely related to a problem in dissipative particle dynamics involving an anharmonic form of particle kinetic energy. In particular, the maximum value of $Y$ reached along the USRSE string, $Y_{\max }$, is analogous to the quasi-particle turning point (at which the quasi-velocity $m=\frac{d Y}{d X}=0$ ). On the USRSE side of this analogy, we note that $Y_{\max }$ plays a significant role in the applications of USRSE for launching spacecraft (Golubović \& Knudsen, 2009; Knudsen \& Golubović, 2014). The $Y_{\max }$ corresponds (via $R_{2, \max }=$ $\lambda Y_{\text {max }}$; see Eq. (6)) to the maximum value of $R_{2}$. This location (apex of the string) plays essential role in the applications of USRSE to launching spacecraft (Golubović \& Knudsen, 2009; Knudsen \& Golubović, 2014) because the local rotating string's speed $\left(\Omega_{R S E} R_{2}\right)$ is at its maximum at the apex.

We will consider Eq. (21) in the form,

$$
\frac{d}{d x} E=-\sqrt{\alpha} m^{2}
$$

with,

$$
E=\frac{1}{2} \ln \left(1+m^{2}\right)+\frac{Y^{2}}{2}=\ln \left(\frac{1}{\cos (\theta(Y))}\right)+\frac{Y^{2}}{2}
$$

Here,

$$
m=\frac{d Y}{d X}=\tan (\theta)
$$

is the slope (relative to $\mathrm{X}$ axis) of the tangent to the string. Our next step will be to consider the quantities $E$, $m$, and the tangent slope angle $\theta$ as functions of $Y$ rather than $X$. For this purpose, in Eq. (23), we write the derivative as $\frac{d}{d X}=\frac{d Y}{d X} \frac{d}{d Y}=m \frac{d}{d Y}$, transforming Eq. (23) into,

$$
\frac{d}{d Y} E=-\sqrt{\alpha} m
$$

By Eq. (24),

$$
m(Y)=\sqrt{e^{2 E(Y)-Y^{2}}-1}
$$

on the left ascending $(m>0)$ string section in Figure 1(a). By Eqs. (26) and (27),

$$
\frac{d}{d Y} E(Y)=-\sqrt{\alpha} \sqrt{e^{2 E(Y)-Y^{2}}-1}
$$

In the following we focus on obtaining an asymptotically exact solution to Eq. (28) in small- $\alpha$ limit. For $\alpha=0$, Eq. (28) is manifestly solvable, yielding $E(Y)=$ const. Therefore, $E(Y)=E(Y=0)=E\left(Y=Y_{\max }\right)$, so, by noting that $\theta(Y)=0$ at the maximum value of $Y=Y_{\max }$, Eq. (24) yields,

$$
E=\ln \left(\frac{1}{\cos (\theta(Y))}\right)+\frac{Y^{2}}{2}=\ln \left(\frac{1}{\cos (\theta(Y=0))}\right)=\frac{\left(Y_{\max }\right)^{2}}{2}
$$

Thus, for $\alpha=0$,

$$
\left(Y_{\max }\right)^{2}=\ln \left(\frac{1}{[\cos (\theta(Y=0))]^{2}}\right)
$$

Therefore, in the $\alpha=0$ case, the maximum value of $Y$ depends only on the value of the local string slope (relative to $\mathrm{X}$ axis) at the string base, $\theta(Y=0)$. 
However, due to gravity, the $\alpha$ value for the USRSE is not zero; see Eq. (9). Moreover, as seen in Figure 1, for the USRSE, the string is tangential to the Earth surface. So, $\theta(Y=0)=90^{\circ}$ for the USRSE case. A $\theta(Y=0)$ value smaller then $90^{\circ}$ would produce an energy barrier (due to gravity) keeping sliding climbers localized on the planet surface. The climber dynamics depicted in Figure 1(a) happens thanks to having $\theta(Y=0)=90^{\circ}$. In addition, with $\theta(Y=0)=90^{\circ}$, the tension forces in the upper and lower string branch in Figure 1 are exactly balanced yielding zero net force exerted on the string's tying point to the Earth, which is obviously a desirable feature. Interestingly, while the gravity induces a nonzero $\alpha$ in the USRSE case, and while the contact angle $\theta(Y=0)$ is exactly $90^{\circ}$, the following discussions show that the value of $Y_{\max }$ is approximately given by an equation of the form of Eq. (30) however with an effective, $\alpha$-dependent contact angle (the angle $\theta_{\text {eff }}$ in Eqs. (57-58) in the following).

To proceed, we change the variable $E(Y)$ into the variable,

$$
Z(Y)=e^{-E(Y)}
$$

This change transforms Eq. (28) into the equation,

$$
\frac{d}{d Y} Z(Y)=\sqrt{\alpha} e^{-Y^{2} / 2} \sqrt{1-Z^{2}(Y) e^{Y^{2}}}
$$

Importantly, as shown in the following, Eq. (32) turns out to be tractable in small- $\alpha$ limit by asymptotic methods (see, e.g., Mathews \& Walker, 1970). Note that, by Eqs. (31) and (24),

$$
Z(Y)=\cos (\theta(Y)) e^{-Y^{2} / 2}
$$

Thus, at the string's apex $\left(Y=Y_{\max }\right.$, where $\left.\theta=0\right)$, one has

$$
Z\left(Y_{\max }\right)=e^{-\left(Y_{\max }\right)^{2} / 2}
$$

while at the string base (at the $Y=0$ contact with the planet),

$$
Z(Y=0)=\cos (\theta(Y=0))
$$

We recall that for the actual USRSE, $\theta(Y=0)=90^{\circ}$, so $Z(Y=0)=0$.

To asymptotically solve Eq. (32) in small- $\alpha$ limit, we turn it into the integral equation,

$$
Z(Y)=Z(0)+\sqrt{\alpha} \int_{0}^{Y} d Y^{\prime} e^{-\left(Y^{\prime}\right)^{2} / 2} \sqrt{1-Z^{2}\left(Y^{\prime}\right) e^{+\left(Y^{\prime}\right)^{2}}}
$$

By expanding the square root on RHS of Eq. (36) by means of binomial formula, and by integrating term by term, we find,

$$
Z(Y)=Z(0)+\sqrt{\alpha}\left[I_{0}(Y)+\sum_{n=1}^{\infty}(-1)^{n}\left(\begin{array}{c}
1 / 2 \\
n
\end{array}\right) I_{n}(Y ;\{Z(*)\})\right]
$$

with $I_{0}(Y)=\int_{0}^{Y} d Y^{\prime} e^{-\left(Y^{\prime}\right)^{2} / 2}$, i.e.,

$$
I_{0}(Y)=\sqrt{\frac{\pi}{2}}-\int_{Y}^{\infty} d Y^{\prime} e^{-\left(Y^{\prime}\right)^{2} / 2}
$$

And

$$
I_{n}(Y ;\{Z(*)\})=\int_{0}^{Y} d Y^{\prime} e^{(2 n-1)\left(Y^{\prime}\right)^{2} / 2}\left[Z\left(Y^{\prime}\right)\right]^{2 n}
$$

for $n=1,2,3, \ldots$. For $Y^{2} \gg 1$, the integral in Eq. (38) is dominated by the lower bound ( $Y^{\prime}$ values just above $Y$ ) and it can be asymptotically calculated, with the result,

$$
I_{0}(Y)=\sqrt{\frac{\pi}{2}}-\frac{e^{-Y^{2} / 2}}{Y}
$$


to the leading order for a large $Y^{2}$. On the other side, the integrals in Eq. (39) are all dominated by the upper bound $\left(Y^{\prime}\right.$ values just below $\left.Y\right)$. These integrals can be thus also asymptotically calculated for $Y^{2} \gg 1$, with the result,

$$
I_{n}(Y ;\{Z(*)\})=\frac{e^{-Y^{2} / 2}}{(2 n-1) Y}\left[e^{Y^{2}} Z^{2}(Y)\right]^{n}=\frac{e^{-Y^{2} / 2}}{(2 n-1) Y}\left[\cos ^{2}(\theta(Y))\right]^{n},
$$

to the leading order for a large $Y^{2}$. See the appendix for discussions of the asymptotic expansions yielding Eqs. (40) and (41). By Eqs. (35), (37), (40), and (41), we find that for $Y^{2} \gg 1$,

$$
Z(Y)=\cos (\theta(Y=0))+\sqrt{\alpha}\left[\sqrt{\frac{\pi}{2}}+\frac{e^{-\frac{Y^{2}}{2}}}{Y} f[Q(Y, Z(Y))]\right]
$$

where

$$
Q(Y, Z(Y))=Z^{2}(Y) e^{Y^{2}} \equiv \cos ^{2}(\theta(Y)),
$$

while the function $f[Q]$ in Eq. (42) is,

$$
f[Q]=-1+\sum_{n=1}^{\infty}\left(\begin{array}{c}
1 / 2 \\
n
\end{array}\right) \frac{(-Q)^{n}}{2 n-1}
$$

As shown in the appendix, the sum in Eq. (44) turns out to be exactly doable, yielding,

$$
f[Q]=-\sqrt{1-Q}-\sqrt{Q} \operatorname{Arccos}(\sqrt{1-Q})
$$

Thus, by Eqs. (42) through (45), we find that, for $Y^{2} \gg 1$,

$$
\cos (\theta(Y)) e^{-Y^{2} / 2}=\cos (\theta(0))+\sqrt{\alpha}\left[\sqrt{\frac{\pi}{2}}-\frac{e^{-\frac{Y^{2}}{2}}}{Y}\left(\sin (\theta(Y))+\cos (\theta(Y))\left(\frac{\pi}{2}-\theta(Y)\right)\right)\right]
$$

Eq. (46) provides the way to obtain important string morphology details in the situations with a large $\left(Y_{\max }\right)^{2}$, such as the value of $\left(Y_{\max }\right)^{2}$ itself. The condition $\left(Y_{\max }\right)^{2} \gg 1$, implicitly assumed in the above asymptotic analysis, is indeed always realized for USRSE in the small- $\alpha$ limit, as self-consistently confirmed in the following. Let us obtain $\left(Y_{\max }\right)^{2}$ by means of Eq. (46) with $Y=Y_{\max }$. Since $\theta\left(Y_{\max }\right)=0$, Eq. (46) reduces to the equation,

$$
e^{-\left(Y_{\max }\right)^{2} / 2}=\cos (\theta(0))+\sqrt{\alpha}\left[\sqrt{\frac{\pi}{2}}-\frac{\pi}{2} \frac{e^{-\left(Y_{\max }\right)^{2} / 2}}{Y_{\max }}\right]
$$

Note that, for $\alpha=0$, Eq. (47) reproduces the exact result in Eq. (30). More than this, Eq. (47) can be iteratively solved for $Y_{\max }$ in small- $\alpha$ limit. For this purpose, we rewrite Eq. (47) as

$$
\left(Y_{\text {max }}\right)^{2}=\ln \frac{1}{\left(\cos (\theta(0))+\sqrt{\frac{\pi}{2} \alpha}\right)^{2}}+2 \ln \left(1+\frac{\pi}{2} \frac{\sqrt{\alpha}}{Y_{\max }}\right)
$$

When $\sqrt{\alpha}$ and $\cos (\theta(0))$ are both small, the first term in RHS of Eq. (48) dominates over the second term, so to the leading order in small $\alpha$,

$$
\left(Y_{\max }\right)^{2}=\ln \frac{1}{\left(\cos (\theta(0))+\sqrt{\frac{\pi}{2} \alpha}\right)^{2}}
$$

To get the leading correction to this result, we insert the result in Eq. (49) into the second term on the RHS of Eq. (48). This yields,

$$
\left(Y_{\max }\right)^{2}=\ln \frac{1}{\left(\cos (\theta(0))+\sqrt{\frac{\pi}{2} \alpha}\right)^{2}}+\pi \frac{\sqrt{\alpha}}{\sqrt{\ln \frac{1}{\left(\cos (\theta(0))+\sqrt{\frac{\pi}{2} \alpha}\right)^{2}}}}
$$

to $O(\sqrt{\alpha})$. For USRSE case, $\cos (\theta(0))=0$, so by Eq. $(50)$, 


$$
\left(Y_{\max }\right)^{2}=\ln \left(\frac{1}{\frac{\pi}{2} \alpha}\right)+\pi \frac{\sqrt{\alpha}}{\left.\sqrt{\ln \left(\frac{1}{\frac{\pi}{2}} \alpha\right.}\right)}=\ln \left(\frac{1}{\frac{\pi}{2} \alpha}\right)\left(1+\pi \frac{\sqrt{\alpha}}{\left[\ln \left(\frac{1}{\frac{\pi}{2} \alpha}\right)\right]^{3 / 2}}\right)
$$

Eq. (51) is the central result of the small- $\alpha$ expansion. It will be used in the following to discuss launching of spacecraft by applying USRSE. Note that in small- $\alpha$ limit the first term on RHS of Eq. (51) actually diverges. The second term on RHS of Eq. (51) is the leading correction which approaches 0 when $\alpha \rightarrow 0$. Thus, for small- $\alpha$, to the leading order,

$$
\left(Y_{\max }\right)^{2} \cong\left(Y_{m}\right)^{2}
$$

with,

$$
\left(Y_{m}\right)^{2}=\ln \left(\frac{1}{\frac{\pi}{2} \alpha}\right)
$$

In view of Eq. (53), the small- $\alpha$ expansion can also be thought as large- $\left(Y_{m}\right)^{2}$ expansion. For example, in terms of $Y_{m}$ defined in Eq. (53) [yielding $\alpha=\frac{2}{\pi} \exp \left(-\left(Y_{m}\right)^{2}\right)$ ], the result in Eq. (51) is expressed as

$$
\left(Y_{\max }\right)^{2}=\left(Y_{m}\right)^{2}+\sqrt{2 \pi} \frac{e^{-\left(Y_{m}\right)^{2} / 2}}{Y_{m}}=\left(Y_{m}\right)^{2}\left(1+\sqrt{2 \pi} \frac{e^{-\left(Y_{m}\right)^{2} / 2}}{Y_{m}^{3}}\right)
$$

By Eq. (54), it is very transparent that, for $\left(Y_{m}\right)^{2} \gg 1$, the leading term $\left[\left(Y_{m}\right)^{2}\right.$ on the RHS of Eq. (54) strongly dominates over the exponentially small correction seen in the second term in Eq. (54). For example, at $Y_{m}=2.5$ (i.e. $\alpha=0.32 \cdot 10^{-2}$ ) the ratio between the second and the first term on RHS of Eqs (54) [i.e., Eq. (51)] is already 0.0146 , and it rapidly decreases below this value with increasing $Y_{m}$, i.e. decreasing $\alpha$. See Figure 2 below.

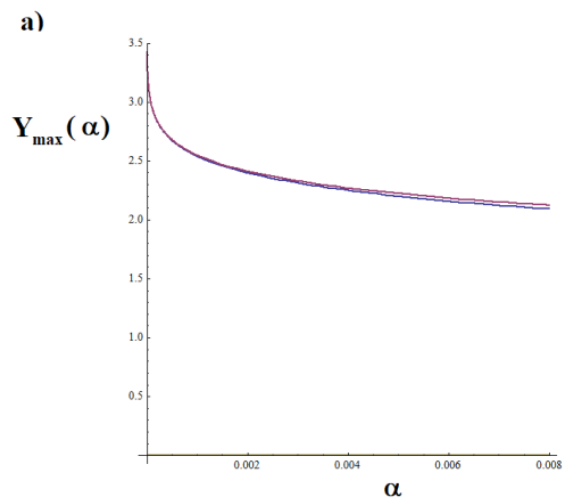

b)

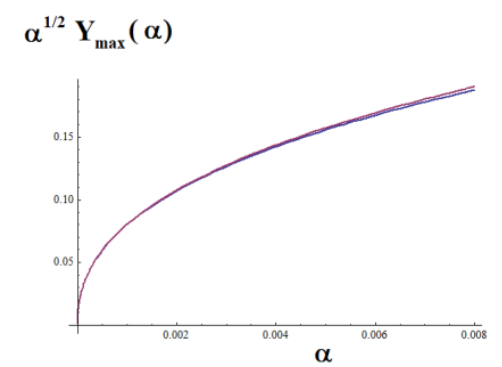

Figure 2. In panel (a), we display $Y_{\max }(\alpha)$ versus $\alpha$. In panel (b), we display $\sqrt{\alpha} Y_{\max }(\alpha)$ versus $\alpha$. In each panel, upper (lower) curves are the results obtained with (without) leading correction included. The displayed results are significant in connection with the results in Eqs. (15) and (19); see the discussions after Eq. (17)

$$
Y_{\max }(\alpha)=\sqrt{\ln \left(\frac{1}{\frac{\pi}{2} \alpha}\right)}\left(1+\frac{\pi}{2} \frac{\sqrt{\alpha}}{\left[\ln \left(\frac{1}{\frac{\pi}{2}}\right)\right]^{3 / 2}}\right)
$$

Let us also note that Eq. (50) can be iteratively inverted to obtain $\alpha$ as a function of $Y_{\max }$, with the result,

$$
\alpha\left(Y_{\max }\right)=\frac{2}{\pi} e^{-\left(Y_{\max }\right)^{2}}\left(1+\sqrt{2 \pi} \frac{e^{-\left(Y_{\max }\right)^{2} / 2}}{Y_{\max }}\right)
$$

Finally, it is interesting to compare the exact result in Eq. (30) obtained for zero $\alpha$ case and a non-zero $\cos (\theta(0))$, with the result in Eqs. (52-53) obtained for the USRSE case with non-zero $\alpha$ and zero $\cos (\theta(0))$. Apparently, 
the USRSE case result can be approximately related to the zero $\alpha$ result Eq. (30) with an effective slope angle $\theta_{\text {eff }}$ at the string base which is given by,

$$
\cos \left(\theta_{\text {eff }}\right)=\sqrt{\frac{\pi}{2} \alpha}
$$

In terms of the effective contact angle $\theta_{\text {eff }}$, the result in Eq. (52-53) can be expressed as,

$$
\left(Y_{\max }\right)^{2} \cong\left(Y_{m}\right)^{2}=\ln \left(\frac{1}{\left[\cos \left(\theta_{e f f}\right)\right]^{2}}\right)
$$

\section{USRSE Morphology and Applications for Spacecraft Launching}

The USRSE apex is the fastest point along the rotating string. Therefore, for spacecraft launching, the most important USRSE morphological element is the $R_{2}$ coordinate of the string apex, $R_{2, \max }$, as well the rotating string speed at the apex (in the non-inertial frame of the rotating planet), $v_{\max }$. To discuss these elements both qualitatively and quantitatively, we will use our results from Secs. 2 and 3. Let us first combine these results into a set of informative formulas. Thus, by combining the results in Eq. (55) with Eqs. (15), (18), and (19), we obtain the results,

$$
\begin{gathered}
\frac{v_{\max }}{v_{1}}=\frac{1}{1-\Delta_{1}} \sqrt{\widetilde{K}} Y_{\max }(\alpha)=\frac{1}{1-\Delta_{1}} \sqrt{\widetilde{K}} \sqrt{\ln \left(\frac{1}{\frac{\pi}{2} \alpha}\right)}\left(1+\frac{\pi}{2} \frac{\sqrt{\alpha}}{\left[\ln \left(\frac{1}{\frac{\pi}{2}}\right)\right]^{3 / 2}}\right) \\
\frac{R_{2, \max }}{R_{E}}=\frac{\widetilde{K}}{1-\Delta_{1}} \sqrt{\alpha} Y_{\max }(\alpha)=\frac{\widetilde{K}}{1-\Delta_{1}} \sqrt{\alpha} \sqrt{\ln \left(\frac{1}{\frac{\pi}{2} \alpha}\right)}\left(1+\frac{\pi}{2} \frac{\sqrt{\alpha}}{\left[\ln \left(\frac{1}{\frac{\pi}{2} \alpha}\right)\right]^{3 / 2}}\right)
\end{gathered}
$$

as well as the results,

$$
\begin{gathered}
\frac{v_{\max }}{v_{1}}=\frac{1}{1-\Delta_{1}} \sqrt{\widetilde{K}} \sqrt{\ln \left(\frac{2}{\pi} \widetilde{K}\left(\frac{T_{1}}{T_{R S E}}\right)^{2}\right)}\left(1+\frac{\pi}{2} \frac{\frac{1}{\sqrt{\widetilde{K}}} \frac{T_{R S E}}{T_{1}}}{\left[\ln \left(\frac{2}{\pi} \widetilde{K}\left(\frac{T_{1}}{T_{R S E}}\right)^{2}\right)\right]^{3 / 2}}\right) \\
\frac{R_{2, \max }}{R_{E}}=\frac{T_{R S E}}{T_{1}} \frac{v_{\max }}{v_{1}}=\frac{1}{1-\Delta_{1}} \sqrt{\widetilde{K}} \frac{T_{R S E}}{T_{1}} \sqrt{\ln \left(\frac{2}{\pi} \widetilde{K}\left(\frac{T_{1}}{T_{R S E}}\right)^{2}\right)}\left(1+\frac{\pi}{2} \frac{\frac{1}{\sqrt{\widetilde{K}}} \frac{T_{R S E}}{T_{1}}}{\left[\ln \left(\frac{2}{\pi} \widetilde{K}\left(\frac{T_{1}}{T_{R S E}}\right)^{2}\right)\right]^{3 / 2}}\right)
\end{gathered}
$$

The results in Eqs. (59) through (62) incorporate both the leading terms and the leading corrections coming from the small $\alpha$ expansion [see Eqs (51) and (54) and the discussions]. These corrections are however small (see Figure 2) and they do not qualitatively affect the results.

It should be stressed that the results Eqs (59)-(62) are valid for any planet, as they express major USRSE properties $v_{\max } / v_{1}$ and $R_{2, \max } / R_{E}$ in terms of the USRSE parameters $\left(\widetilde{K}, \frac{T_{R S E}}{T_{1}}, \Delta_{1}\right)$. We recall that $\Delta_{1}=\left(\frac{T_{1}}{T_{\text {day }}}\right)^{2}$ is the property of the planet. Due to its smallness, this parameter is somewhat secondary; in the plots in Figure we used the Earth's $\Delta_{1}=0.00346$. The results in Eqs. (59)-(62) are displayed in Figure 3. As seen in Figure 3, major qualitative feature of $\frac{v_{\max }}{v_{1}}$ is that it diverges in the small- $\alpha$ limit, i.e., in the small $\frac{T_{R S E}}{T_{1}}$ limit. This result, in connection with Eq. (16), is fundamental since it shows that $v_{\max }$ will generally exceed the $1^{\text {st }}$ cosmic speed $v_{1}$, for any $\widetilde{K}$ value, provided $\alpha$, i.e., $\frac{T_{R S E}}{T_{1}}$ is small enough, as documented in Figures 3(a-d). Thus, USRSE system can be used for spacecraft launching provided $\frac{T_{R S E}}{T_{1}}$ is small enough. Another general feature of USRSE morphology evidenced in Figures 3(e,f) is that with decreasing $\frac{T_{R S E}}{T_{1}}$ (i.e., decreasing $\alpha$ ), USRSE linear sizes, 
such as $R_{2, \max }$ decrease relative to the planet radius $R_{E}$. This self-consistently justifies the expansion yielding the Eqs. (3), (4), end, eventually, Eq. (8) which is central to this study. Another set of insightful relations is obtained by combining Eq. (56) with Eqs. (18) and (19). This yields the results,

$$
\alpha=\frac{2}{\pi} \exp \left[-\frac{\left(1-\Delta_{1}\right)^{2}}{\widetilde{K}}\left(\frac{v_{\max }}{v_{1}}\right)^{2}\right]\left(1+\sqrt{2 \pi} \frac{\exp \left[-\frac{\left(1-\Delta_{1}\right)^{2}}{2 \widetilde{v}}\left(\frac{v_{\max }}{v_{1}}\right)^{2}\right]}{\frac{1-\Delta_{1} v_{\max }}{v_{1}}}\right)
$$

And

$$
\frac{T_{R S E}}{T_{1}}=\sqrt{\alpha \widetilde{K}}=\sqrt{\frac{2}{\pi}} \sqrt{\widetilde{K}} \exp \left[-\frac{\left(1-\Delta_{1}\right)^{2}}{2 \widetilde{K}}\left(\frac{v_{\max }}{v_{1}}\right)^{2}\right]\left(1+\sqrt{\frac{\pi}{2}} \frac{\exp \left[-\frac{\left(1-\Delta_{1}\right)^{2}}{2 \widetilde{K}}\left(\frac{v_{\max }}{v_{1}}\right)^{2}\right]}{\frac{1-\Delta_{1} v_{\max }}{v_{1}}}\right)
$$

a)

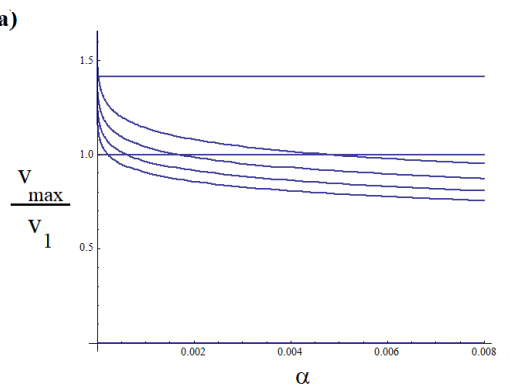

c)

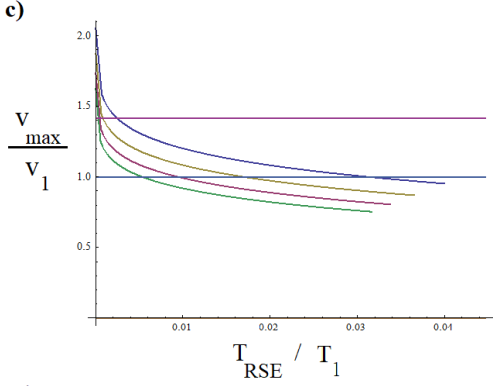

e)

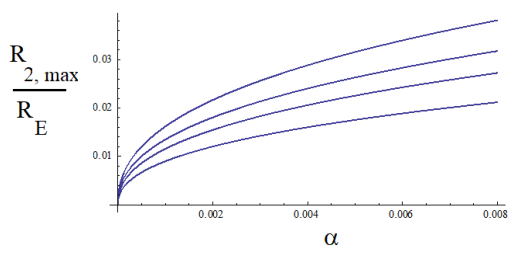

b)

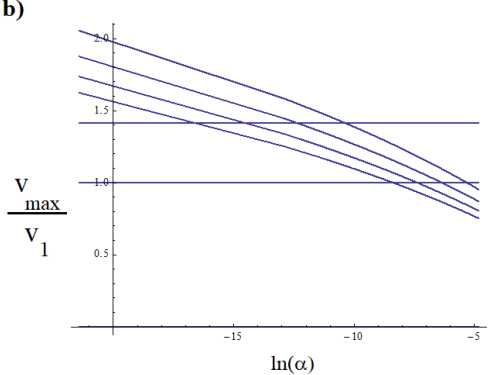

d)

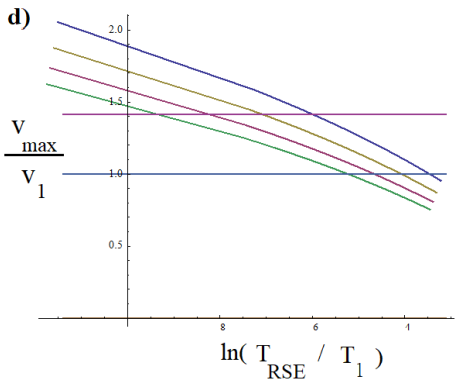

f)

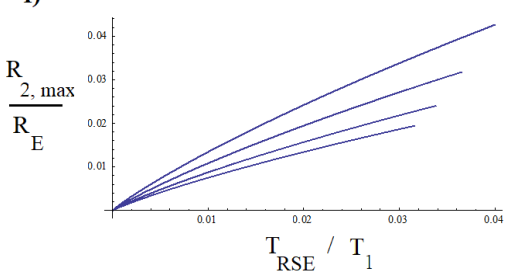

Figure 3. Panels (a) - (d), the results for $v_{\max } / v_{1}$ versus $\alpha$ [Eq. (59)] and versus $\frac{T_{R S E}}{T_{1}}$ [Eq. (61)]. In each panel, the four curves are (from top to bottom) for the cases $\widetilde{K}=\frac{1}{5}, \frac{1}{6}, \frac{1}{7}, \frac{1}{8}$. The two horizontal lines are at $v_{\max }=v_{1}=1^{\text {st }}$ cosmic speed, and $v_{\max }=v_{2}=\sqrt{2} \quad v_{1}=2^{\text {nd }}$ cosmic speed. Panels $(\mathrm{e})-(\mathrm{f})$, the results for $R_{2, \max } / R_{E}$ versus $\alpha$ [Eq. (60)] and versus $\frac{T_{R S E}}{T_{1}}$ [Eq. (62)]. In each panel, the four curves are (from top to bottom) for the cases $\widetilde{K}=\frac{1}{5}, \frac{1}{6}, \frac{1}{7}$, and $\frac{1}{8}$ 
a)

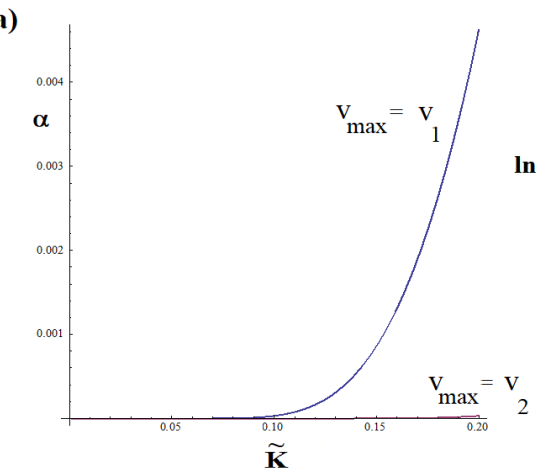

c)

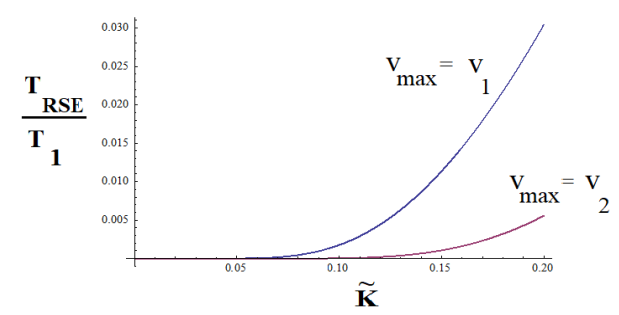

b)

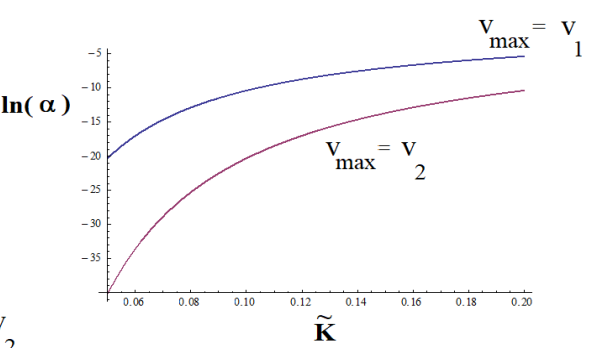

d)

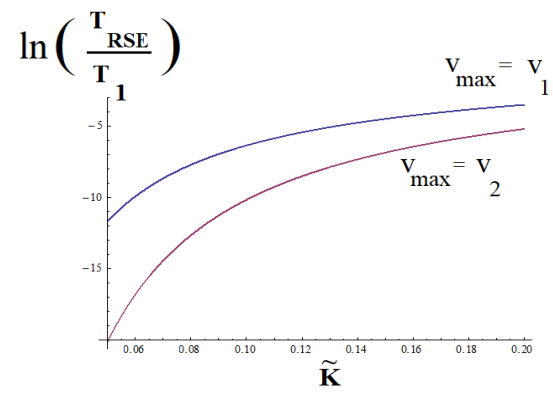

Figure 4. Plots of the results in Eqs. (63) and (64) for two cases: $v_{\max }=v_{1}=1^{\text {st }}$ cosmic speed, and $v_{\max }=$ $v_{2}=\sqrt{2} \quad v_{1}=2^{\text {nd }}$ cosmic speed

The results in Eqs. (63) and (64) (plotted in Figure 4) provide answers to a practically important question: What should be the value of the USRSE parameters $\frac{T_{R S E}}{T_{1}}$ to achieve a desired rotational speed at the apex, $v_{\text {max }}$ ? From Figure 4 , it is clear that to achieve a significant $v_{\max }$, such as $1^{\text {st }}$ or $2^{\text {nd }}$ cosmic speed, it requires using very small $\frac{T_{R S E}}{T_{1}}$ if the value of $\widetilde{K}$ is small. For such values of $\frac{v_{\max }}{v_{1}}$ and for a small $\widetilde{K}$, one can drop the leading correction terms in Eq. (64). This yields the simple result,

$$
\frac{T_{R S E}}{T_{1}}=\sqrt{\frac{2}{\pi}} \sqrt{\widetilde{K}} \exp \left[-\frac{\left(1-\Delta_{1}\right)^{2}}{2 \widetilde{K}}\left(\frac{v_{\max }}{v_{1}}\right)^{2}\right]
$$

Clearly by Eq. (65), to achieve $v_{\max }=v_{1}=1^{\text {st }}$ cosmic speed or $v_{\max }=v_{2}=\sqrt{2} v_{1}=2^{\text {nd }}$ cosmic speed with a small $\widetilde{K}$, it would take using USRSE with a very small $\frac{T_{R S E}}{T_{1}}$.

Let us finally address spacecraft launching by means of USRSE. There are several possible scenarios of spacecraft launching by using USRSE (see Knudsen \& Golubović, 2014). With sliding friction present, sliding climbers starting at the planet surface would eventually stop near the RSE point minimizing the effective potential Eq. (2). In the small- $\alpha$ limit, the stopping point is very close to USRSE apex, as evidenced by equipotentials displayed in Figure 1(a). Thus, due to sliding friction, a sliding climber carrying a spacecraft eventually stops at the apex where it can release the carried spacecraft into outer space. Let us consider this type of spacecraft launching from the apex of the lower USRSE branch when it is in the plane of Figure 1(a). The spacecraft is initially at rest with respect to the string at the apex, and then it is released into the direction of the planet rotation (into the Figure 1 plane). The released spacecraft speed in the inertial frame will be then,

$$
v_{\text {released }}=v_{\text {max }}+\Delta v
$$

with the $\Delta v$ coming from the planet rotation,

$$
\Delta v=\Omega_{E} R_{1, \text { apex }}=\Omega_{E}\left(R_{E}+x_{\text {apex }}\right)
$$

Here, the x-coordinate of the apex, 


$$
x_{\text {apex }}=\lambda X_{\text {apex }}
$$

where $\lambda$ is the USRSE characteristic length scale (Eqs. (7) and (14)), and,

$$
X_{\text {apex }}=\frac{\pi}{2 Y_{m}(\alpha)}=\frac{\pi}{2 \sqrt{\ln \left(\frac{1}{\frac{\pi}{2} \alpha}\right)}}
$$

See the appendix for the derivation of Eq. (69). By Eqs. (68-69) combined with Eq. (14),

$$
\frac{x_{\text {apex }}}{R_{E}}=\frac{\widetilde{K}}{1-\Delta_{1}} \frac{\frac{\pi}{2} \sqrt{\alpha}}{\sqrt{\ln \left(\frac{1}{\frac{\pi}{2}} \alpha\right)}}
$$

Thus, the contribution of the planet rotation to the released spacecraft velocity satisfies the relation,

$$
\frac{\Delta v}{v_{1}}=\frac{\Omega_{E} R_{E}}{v_{1}}\left(1+\frac{x_{\text {apex }}}{R_{E}}\right)=\frac{T_{1}}{T_{\text {day }}}\left(1+\frac{x_{\text {apex }}}{R_{E}}\right)=\frac{T_{1}}{T_{\text {day }}}\left(1+\frac{\widetilde{K}}{1-\Delta_{1}} \frac{\frac{\pi}{2} \sqrt{\alpha}}{\sqrt{\ln \left(\frac{1}{\frac{\pi}{2} \alpha}\right)}}\right)
$$

We recall that for the Earth $\frac{T_{1}}{T_{d a y}}=\sqrt{\Delta_{1}}=0.0588$. By the above and Eq. (59), we get the relation,

$$
\frac{v_{\text {relased }}}{v_{1}}=\frac{v_{\text {max }}}{v_{1}}+\frac{\Delta v}{v_{1}}=\frac{v_{\text {max }}}{v_{1}}+\frac{T_{1}}{T_{\text {day }}}\left(1+\frac{x_{\text {apex }}}{R_{E}}\right)=\frac{1}{1-\Delta_{1}} \sqrt{\widetilde{K}} \sqrt{\ln \left(\frac{1}{\frac{\pi}{2} \alpha}\right)}\left(1+\frac{\pi}{2} \frac{\sqrt{\alpha}}{\left[\ln \left(\frac{1}{\frac{\pi}{2} \alpha}\right]^{3 / 2}\right.}\right)+\frac{T_{1}}{T_{\text {day }}}\left(1+\frac{\widetilde{K}}{1-\Delta_{1}} \frac{\frac{\pi}{2} \sqrt{\alpha}}{\sqrt{\ln \left(\frac{1}{\frac{\pi}{2}}\right)}}\right) \text { (72) }
$$

which compares the released spacecraft velocity with the $1^{\text {st }}$ cosmic speed at the planet surface, $v_{1}$. However, to infer the actual effect of the launch, one needs to compare $v_{\text {released }}$ with the first cosmic speed at the position of the apex relative to the planet center, $\left(R_{1, \text { apex }}=R_{E}+x_{\text {apex }}, R_{2, \max }\right)$, given by,

$$
v_{1, \text { apex }}=\left(\frac{R_{E}}{R_{\text {apex }}}\right)^{1 / 2} v_{1}=\frac{v_{1}}{\left[\left(1+\frac{x_{\text {apex }}}{R_{E}}\right)^{2}+\left(\frac{R_{2, \text { max }}}{R_{E}}\right)^{2}\right]^{1 / 4}}
$$

With this we have

$$
\begin{aligned}
& \frac{v_{\text {released }}}{v_{1, \text { apex }}}=\left[\left(1+\frac{x_{\text {apex }}}{R_{E}}\right)^{2}+\left(\frac{R_{2, \text { max }}}{R_{E}}\right)^{2}\right]^{1 / 4} \frac{v_{\text {released }}}{v_{1}}=\left[\left(1+\frac{x_{\text {apex }}}{R_{E}}\right)^{2}+\left(\frac{R_{2, \text { max }}}{R_{E}}\right)^{2}\right]^{1 / 4}\left[\frac{v_{\text {max }}}{v_{1}}+\frac{T_{1}}{T_{\text {day }}}\left(1+\frac{x_{\text {apex }}}{R_{E}}\right)\right] \cong \\
& \left(1+\frac{x_{\text {apex }}}{R_{E}}\right)^{1 / 2}\left[\frac{v_{\text {max }}}{v_{1}}+\frac{T_{1}}{T_{\text {day }}}\left(1+\frac{x_{\text {apex }}}{R_{E}}\right)\right] \cong\left(1+\frac{1}{2} \frac{x_{\text {apex }}}{R_{E}}\right) \frac{v_{\text {max }}}{v_{1}}+\frac{T_{1}}{T_{\text {day }}}\left(1+\frac{3}{2} \frac{x_{\text {apex }}}{R_{E}}\right)
\end{aligned}
$$

valid to $\mathrm{O}(\sqrt{\alpha})$. Using here Eqs. (59) and (70), we obtain, to $\mathrm{O}(\sqrt{\alpha})$,

$$
\begin{gathered}
\frac{v_{\text {released }}}{v_{1, \text { apex }}} \cong\left(1+\frac{1}{2} \frac{\widetilde{K}}{1-\Delta_{1}} \frac{\frac{\pi}{2} \sqrt{\alpha}}{\left.\sqrt{\ln \left(\frac{1}{\frac{\pi}{2}}\right)}\right)}\right) \frac{1}{1-\Delta_{1}} \sqrt{\widetilde{K}} \sqrt{\ln \left(\frac{1}{\frac{\pi}{2} \alpha}\right)}\left(1+\frac{\frac{\pi}{2} \sqrt{\alpha}}{\left[\ln \left(\frac{1}{\frac{\pi}{2} \alpha}\right)^{3 / 2}\right.}\right)+\frac{T_{1}}{T_{\text {day }}}\left(1+\frac{3}{2} \frac{\widetilde{K}}{1-\Delta_{1}} \frac{\frac{\pi}{2} \sqrt{\alpha}}{\sqrt{\ln \left(\frac{1}{\frac{\pi}{2} \alpha}\right)}}\right) \cong \\
\frac{1}{1-\Delta_{1}} \sqrt{\widetilde{K}} \sqrt{\ln \left(\frac{1}{\frac{\pi}{2} \alpha}\right)}\left(1+\frac{\frac{\pi}{2} \sqrt{\alpha}}{\left[\ln \left(\frac{1}{\frac{\pi}{2} \alpha}\right)\right]^{3 / 2}}+\frac{1}{2} \frac{\widetilde{K}}{1-\Delta_{1}} \frac{\frac{\pi}{2} \sqrt{\alpha}}{\sqrt{\ln \left(\frac{1}{\frac{\pi}{2} \alpha}\right)}}\right)+\frac{T_{1}}{T_{\text {day }}}\left(1+\frac{3}{2} \frac{\widetilde{K}}{1-\Delta_{1}} \frac{\frac{\pi}{2} \sqrt{\alpha}}{\sqrt{\ln \left(\frac{1}{\frac{\pi}{2} \alpha}\right)}}\right)
\end{gathered}
$$

valid to $\mathrm{O}(\sqrt{\alpha})$. Combining Eq. (75) with Eq. (18), we also obtain, 


$$
\begin{gathered}
\frac{\mathrm{v}_{\text {released }}}{\mathrm{v}_{1, \text { apex }}}=\frac{1}{1-\Delta_{1}} \sqrt{\widetilde{\mathrm{K}}} \sqrt{\ln \left(\frac{2}{\pi} \widetilde{\mathrm{K}}\left(\frac{\mathrm{T}_{1}}{\mathrm{~T}_{\mathrm{RSE}}}\right)^{2}\right)}\left(1+\frac{\frac{\pi 1 \mathrm{~T}_{\mathrm{RSE}}}{2 \sqrt{\widetilde{\mathrm{K}}} \mathrm{T}_{1}}}{\left[\ln \left(\frac{2}{\pi} \widetilde{\mathrm{K}}\left(\frac{\mathrm{T}_{1}}{\mathrm{~T}_{\mathrm{RSE}}}\right)^{2}\right)\right]^{3 / 2}}+\frac{1}{2} \frac{1}{1-\Delta_{1}} \frac{\frac{\pi}{2} \sqrt{\mathrm{K}} \frac{\widetilde{\mathrm{R}} \text { RSE }}{\mathrm{T}_{1}}}{\sqrt{\ln \left(\frac{2}{\pi} \widetilde{\mathrm{K}}\left(\frac{\mathrm{T}_{1}}{\mathrm{~T}_{\mathrm{RSE}}}\right)^{2}\right)}}\right)+\frac{\mathrm{T}_{1}}{\mathrm{~T}_{\text {day }}}(1+ \\
\left.\frac{3}{2} \frac{1}{1-\Delta_{1}} \frac{\frac{\pi}{2} \sqrt{\widetilde{\mathrm{K}}} \frac{\mathrm{T}_{\mathrm{RSE}}}{\mathrm{T}_{1}}}{\sqrt{\ln \left(\frac{2}{\pi} \widetilde{\mathrm{K}}\left(\frac{\mathrm{T}_{1}}{\mathrm{~T}_{\mathrm{RSE}}}\right)^{2}\right)}}\right)
\end{gathered}
$$

a)

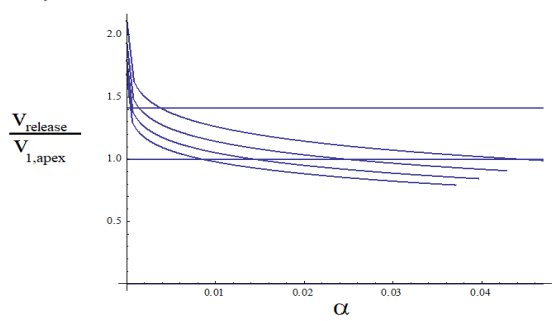

c)

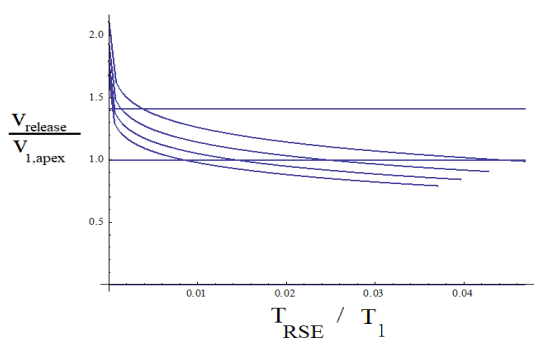

b)

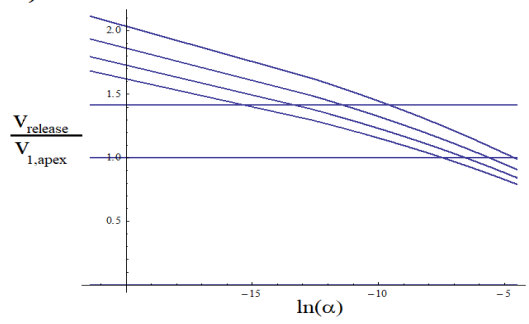

d)

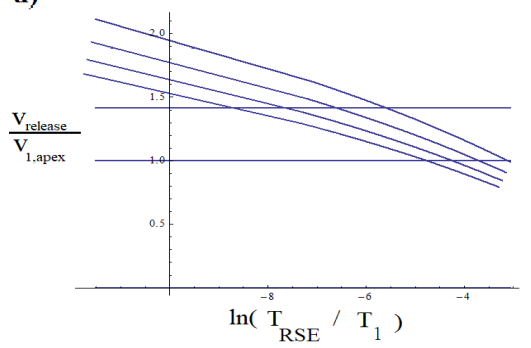

Figure 5. Launching spacecraft by releasing it from USRSE apex into the direction of the planet rotation: the four panels show the results for $v_{\text {release }} / v_{1, \text { apex }}$ versus $\alpha$ [Eq. (75)] and versus $\frac{T_{R S E}}{T_{1}}$ [Eq. (76)]. In each panel, the four curves are (from top to bottom) for the cases $\widetilde{K}=\frac{1}{5}, \frac{1}{6}, \frac{1}{7}, \frac{1}{8}$. The two horizontal lines are at $v_{\text {release }}=$ $v_{1, \text { apex }}=1^{\text {st }}$ cosmic speed at the apex, and $v_{\text {release }}=v_{2, \text { apex }}=\sqrt{2} v_{1, \text { apex }}=2^{\text {nd }}$ cosmic speed at the USRSE apex position

In Figure 5, we display the results in Eqs. (75) and (76) for the case of the Earth, with $\frac{T_{1}}{T_{d a y}}=\sqrt{\Delta_{1}}=0.0588$. From the figure one can see that achieving significant cosmic velocities at small values of $\widetilde{K}$ requires using small values of $\frac{T_{R S E}}{T_{1}}$. In this limit, Eq. (76) can be approximated by

$$
\frac{v_{\text {released }}}{v_{1, \text { apex }}}=\frac{1}{1-\Delta_{1}} \sqrt{\widetilde{K}} \sqrt{\ln \left(\frac{2}{\pi} \widetilde{K}\left(\frac{T_{1}}{T_{R S E}}\right)^{2}\right)}+\frac{T_{1}}{T_{\text {day }}}
$$

yielding the result,

$$
\frac{T_{R S E}}{T_{1}}=\sqrt{\frac{2}{\pi}} \sqrt{\widetilde{K}} \exp \left[-\frac{\left(1-\Delta_{1}\right)^{2}}{2 \widetilde{K}}\left(\frac{v_{\text {release }}}{v_{1, \text { apex }}}-\frac{T_{1}}{T_{\text {day }}}\right)^{2}\right]
$$

Clearly by Eq. (78), to achieve $v_{\text {release }}=v_{1, \text { apex }}=1^{\text {st }}$ cosmic speed (at apex) or $v_{\text {release }}=v_{2, \text { apex }}=$ $\sqrt{2} v_{1, \text { apex }}=2^{\text {nd }}$ cosmic speed (at apex) with a small $\widetilde{K}$, it would take using USRSE with a very small $\frac{T_{R S E}}{T_{1}}$. 


\section{Acknowledgments}

We thank WVHTC and NASA for the sub-grant WVHTC-W-NASA-IR-07-1367 of the Grant NNX06AE557: Innovative Research for Next Generation Space Exploration.

\section{Conflict of interests}

The authors declare that there is no conflict of interests regarding the publication of this paper.

\section{References}

Edwards, B. C., \& Westling, E. A. (2003). The Space Elevator: A Revolutionary Earth-to-Space Transportation System. USA: BC Edwards.

Fitzgibbons, T. C., Guthrie, M., En-shi Xu, E., Crespi, V. H., Davidowski, S. K., Cody, G. D., Alem, N., \& Badding, J. V. (2014). Benzene-derived carbon nanothreads. Nature Materials, 14, 43-47.

Golubović, L., \& Knudsen, S. (2009). Classical and statistical mechanics of celestial scale spinning strings: Rotating space elevators. Europhysics Letters, 86(3), 34001.

Kardar, M. (2007). Statistical Physics of Fields. Cambridge, UK: Cambridge University Press.

Knudsen, S., \& Golubović, L. (2014). Rotating space elevators: Physics of celestial scale spinning strings. European Physical Journal Plus, 129, 242.

Knudsen, S., \& Golubović, L. (2015). Physics of untied rotating space elevators. European Physical Journal Plus, 130, 243.

Knudsen, S., \& Golubović, L. (2016). Modeling the physics of sliding objects for rotating space elevators. European Physical Journal Plus, 130, 242.

Mathews, J., \& Walker, R. L. (1970). Mathematical Methods of Physics. USA: Addison-Wesley Publishing Co.

Nelson, D. R. (2002). Defects and Geometry in Condensed Matter Physics. Cambridge, UK: Cambridge University Press.

Nelson, D. R., Piran, T., \& Weinberg, S. (Eds.). (1988). Statistical Mechanics of Membranes and Surfaces. Singapore: World Scientific.

Pearson, J. (1975). The Orbital Tower: A Spacecraft Launcher Using the Earth's Rotational Energy. Acta Astronautica, 2, 785-799.

Yu, M. F., Files, B. S., Arepalli, S., \& Ruoff, R. S. (2000). Tensile Loading of Ropes of Single Wall Carbon Nanotubes and their Mechanical Properties. Physical Review Letters, 84, 5552-5555.

Yu, M. F., Lourie, O., Dyer, M. J., Moloni, K., Kelly, T. F., \& Ruoff, R. S. (2000). Strength and breaking mechanism of multiwalled carbon nanotubes under tensile load. Science, 287, 637-640.

\section{Appendix}

(I) Mathematical details aiding the derivation of Eq. (42). To calculate the sum in Eq. (44) we rewrite it as

$$
f[Q]=-1+\sqrt{Q} h[Q]
$$

with

$$
\mathrm{h}[\mathrm{Q}]=\sum_{\mathrm{n}=1}^{\infty}\left(\begin{array}{c}
1 / 2 \\
\mathrm{n}
\end{array}\right) \frac{(-1)^{\mathrm{n}} \mathrm{Q}^{\mathrm{n}-\frac{1}{2}}}{2\left(\mathrm{n}-\frac{1}{2}\right)}
$$

Next, note that,

$$
\frac{\mathrm{d}}{\mathrm{dQ}} \mathrm{h}[\mathrm{Q}]=\frac{1}{\mathrm{Q}^{3 / 2}} \sum_{\mathrm{n}=1}^{\infty}\left(\begin{array}{c}
1 / 2 \\
\mathrm{n}
\end{array}\right) \frac{(-1)^{\mathrm{n}} \mathrm{Q}^{\mathrm{n}}}{2}=\frac{1}{2 \mathrm{Q}^{3 / 2}}[\sqrt{1-\mathrm{Q}}-1]
$$

where the last step employed binomial formula. By Eq. (80), $h(0)=0$, so, by Eq. (81),

$$
\mathrm{h}[\mathrm{Q}]=\int_{0}^{\mathrm{Q}} \mathrm{dQ}^{\prime} \frac{1}{2\left(\mathrm{Q}^{\prime}\right)^{3 / 2}}\left[\sqrt{1-\mathrm{Q}^{\prime}}-1\right]
$$

Anti-derivative needed to perform this integration is, 


$$
J[Q]=\int d Q \frac{1}{2 Q^{\frac{3}{2}}}[\sqrt{1-Q}-1]=\frac{1-\sqrt{1-Q}}{\sqrt{Q}}+\operatorname{Arcsin}(\sqrt{1-Q})
$$

as can be verified by differentiation. By Eqs. (82) and (83),

$$
\mathrm{h}[\mathrm{Q}]=\mathrm{J}[\mathrm{Q}]-\mathrm{J}[0]=\left[\frac{1-\sqrt{1-Q}}{\sqrt{\mathrm{Q}}}+\operatorname{Arcsin}(\sqrt{1-Q})\right]-\frac{\pi}{2}=\frac{1-\sqrt{1-Q}}{\sqrt{\mathrm{Q}}}-\operatorname{Arccos}(\sqrt{1-\mathrm{Q}})
$$

By Eqs. (84) and (79), we finally obtain

$$
f[Q]=-\sqrt{1-Q}-\sqrt{Q} \operatorname{Arccos}(\sqrt{1-Q})
$$

as stated in Eq. (45). QED

(II) Asymptotic evaluation of the integrals in Eqs. (38) and (39), yielding the results in Eqs. (40) and (41).

The $I_{0}(Y)$ in Eq. (38) is the standard error-function, and Eq. (40) displays the leading order terms of its asymptotic expansion for large $Y$ (see, Mathews \& Walker, 1974). The correction to the result in Eq. (40) is $\sim e^{-Y^{2} / 2} / Y^{3}$ which is subdominant to the term displayed in Eq. (40). Similar conclusion also applies to the integral in Eq. (39) yielding the result displayed in Eq. (41). To asymptotically evaluate this integral, we change the integration variable $Y^{\prime}$ into the variable $\rho$ such that $Y^{\prime}=Y-\frac{\rho}{(2 n-1) Y}$. This transforms the integral in Eq. (39) into,

$$
\mathrm{I}_{\mathrm{n}}(\mathrm{Y} ;\{\mathrm{Z}(*)\})=\frac{\mathrm{e}^{-\mathrm{Y}^{2} / 2}}{(2 \mathrm{n}-1) \mathrm{Y}}\left[\mathrm{e}^{\mathrm{Y}^{2}} \mathrm{Z}^{2}(\mathrm{Y})\right]^{\mathrm{n}} \mathrm{J}_{\mathrm{n}}(\mathrm{Y})
$$

with,

$$
J_{n}(Y)=1-e^{-(2 n-1) Y^{2}}+J_{n}^{(1)}(Y)-J_{n}^{(2)}(Y)-J_{n}^{(3)}(Y)
$$

where,

$$
\begin{gathered}
\mathrm{J}_{\mathrm{n}}^{(1)}(\mathrm{Y})=\int_{0}^{(2 \mathrm{n}-1) \mathrm{Y}^{2}} \mathrm{~d} \rho \mathrm{e}^{-\rho}\left[\exp \left(\frac{\rho^{2}}{2(2 \mathrm{n}-1) \mathrm{Y}^{2}}\right)-1\right] \\
J_{n}^{(2)}(Y)=\int_{0}^{(2 n-1) Y^{2}} d \rho e^{-\rho}\left[1-\left(\frac{Z\left(Y-\frac{\rho}{(2 n-1) Y}\right)}{Z(Y)}\right)^{2 n}\right] \\
J_{n}^{(3)}(Y)=\int_{0}^{(2 n-1) Y^{2}} d \rho e^{-\rho}\left[\exp \left(\frac{\rho^{2}}{2(2 n-1) Y^{2}}\right)-1\right]\left[1-\left(\frac{Z\left(Y-\frac{\rho}{(2 n-1) Y}\right)}{Z(Y)}\right)^{2 n}\right]
\end{gathered}
$$

Note that all integrands in Eqs. (88) through (90) approach zero in large-Y limit. This, together with Eq. (87), signals that the value of $J_{n}(Y)$ asymptotically approaches 1 in the large-Y limit, that is, the value of $I_{n}(Y ;\{Z(*)\})$ in Eq. (86) approaches the result stated in Eq. (41). Let us examine the small corrections to this result. In the large-Y limit, asymptotic expansions for the integrals in Eqs. (87) through (90) can be generated by expanding the integrands therein in powers of $\frac{\rho}{(2 n-1) Y}$. This yields, for large-Y,

$$
\begin{gathered}
J_{n}^{(1)}(Y)=\int_{0}^{\infty} d \rho e^{-\rho}\left[\frac{\rho^{2}}{2(2 n-1)^{2} Y^{2}}\right]=\frac{1}{(2 n-1) Y^{2}} \\
J_{n}^{(2)}(Y)=\int_{0}^{\infty} d \rho e^{-\rho}\left[\frac{2 n}{2 n-1} \frac{Z^{\prime}(Y)}{Y Z(Y)} \rho\right]=\frac{2 n}{2 n-1} \frac{Z^{\prime}(Y)}{Y Z(Y)} \\
J_{n}^{(3)}(Y)=\int_{0}^{\infty} d \rho e^{-\rho}\left[\frac{\rho^{2}}{2(2 n-1)^{2} Y^{2}}\right]\left[\frac{2 n}{2 n-1} \frac{Z^{\prime}(Y)}{Y Z(Y)} \rho\right]=\frac{6 n}{(2 n-1)^{3}} \frac{Z^{\prime}(Y)}{Y^{3} Z(Y)}
\end{gathered}
$$


To complete the calculation, we need to obtain the value of the logarithmic derivative $\frac{Z^{\prime}(Y)}{Z(Y)}$ for large-Y. By Eq. (32), $\frac{Z^{\prime}(Y)}{Z(Y)}=\sqrt{\alpha} e^{-Y^{2} / 2} \sqrt{\frac{1}{Z^{2}(Y)}-e^{Y^{2}}}$, while by Eq. (42) [for the USRSE case, with $\cos (\theta(0))=0$ ], for $Y^{2} \gg 1$, one has $Z(Y) \cong \sqrt{\frac{\pi}{2} \alpha}$, with $\alpha$ expressible as $\alpha=\frac{2}{\pi} e^{-Y_{\max }^{2}}$; see Eqs. (52-53). We thus find,

$$
\frac{Z^{\prime}(Y)}{Z(Y)}=\sqrt{\frac{2}{\pi}} e^{-Y^{2} / 2} \sqrt{1-e^{-\left(Y_{\max }^{2}-Y^{2}\right)}}
$$

for $Y^{2} \gg$ 1. Thus, by Eqs. (94), in combination with the results in Eqs. (92) and (93), we find

$$
\begin{gathered}
J_{n}^{(2)}(Y)=\frac{2 n}{2 n-1} \frac{1}{Y} \sqrt{\frac{2}{\pi}} e^{-Y^{2} / 2} \sqrt{1-e^{-\left(Y_{\max }^{2}-Y^{2}\right)}} \\
J_{n}^{(3)}(Y)=\frac{6 n}{(2 n-1)^{3}} \frac{1}{Y^{3}} \sqrt{\frac{2}{\pi}} e^{-Y^{2} / 2} \sqrt{1-e^{-\left(Y_{\max }^{2}-Y^{2}\right)}}
\end{gathered}
$$

Overall, by Eq. (91), we see that $J_{n}^{(1)}(Y) \sim \frac{1}{Y^{2}}$, while, by Eqs. (95) and (96), $J_{n}^{(2)}(Y) \sim \frac{1}{Y} e^{-Y^{2} / 2}$ and $J_{n}^{(3)}(Y) \sim \frac{1}{Y^{3}} e^{-Y^{2} / 2}$, for $Y \gg 1$. Thus, the value of $J_{n}(Y)$ in Eq. (87) converges to 1 in the large- $Y$ limit. Therefore, by Eq. (86), the value of $I_{n}(Y ;\{Z(*)\})$ converges to the result stated in Eq. (21). QED (III) Derivation of Eq. (69) for the X-coordinate of apex, stating that $X_{\text {apex }}=\frac{\pi}{2 Y_{m}}$ in the small- $\alpha$ limit. By Eq. (46) [with $\cos (\theta(0))=0$ ] and Eqs. (52), (53) and (57), one has, to the leading order for small $\alpha$, i.e. large $Y_{m}^{2}$,

$$
\cos (\theta(Y)) e^{-Y^{2} / 2}=\sqrt{\frac{\pi}{2} \alpha}=\cos \left(\theta_{\text {eff }}\right)=e^{-Y_{m}^{2} / 2}
$$

for $Y>O(1)$, i.e. $\theta \in\left[0, \theta_{e f f}\right]$. Next, by geometry,

$$
X_{\text {apex }}=\int_{0}^{X_{\text {apex }}} d X=\int_{0}^{Y_{m}} d Y \frac{d X}{d Y}=\int_{0}^{Y_{m}} d Y \frac{1}{\tan (\theta)}=-\int_{0}^{\pi / 2} d \theta \frac{d Y}{d \theta} \frac{1}{\tan (\theta)}
$$

By Eq. (97), one easily finds that,

$$
\frac{\mathrm{d} Y}{\mathrm{~d} \theta}=\frac{-\tan (\theta)}{\sqrt{\mathrm{Y}_{\mathrm{m}}^{2}+\ln \left[\cos ^{2}(\theta)\right]}}
$$

in the range $Y>O(1)$, i.e. $\theta \in\left[0, \theta_{e f f}\right]$, which dominates the integral Eq. (98). Thus, by Eqs. (98) and (99), to the leading order for small- $\alpha$, i.e., large $Y_{m}$,

$$
X_{\text {apex }}=\int_{0}^{\theta_{\text {eff }}} d \theta \frac{\tan (\theta)}{\sqrt{Y_{m}^{2}+\ln \left[\cos ^{2}(\theta)\right]}} \frac{1}{\tan (\theta)}=\frac{1}{Y_{m}} \int_{0}^{\theta_{e f f}} d \theta \frac{1}{\sqrt{1+\frac{1}{Y_{m}^{2}} \ln \left[\cos ^{2}(\theta)\right]}}
$$

By Eq. (100), for a large $Y_{m}^{2}$ (whence, by Eq. (97), $\theta_{\text {eff }} \rightarrow \pi / 2$ ), one obtains the asymptotic result,

$$
X_{\text {apex }}(\alpha)=\frac{1}{Y_{m}} \int_{0}^{\pi / 2} d \theta=\frac{\pi}{2 Y_{m}(\alpha)}=\frac{\pi}{2 \sqrt{\ln \left(\frac{1}{\frac{\pi}{2} \alpha}\right)}}
$$

QED.

Note that $X_{\text {apex }}(\alpha) \rightarrow 0$ in the small $\alpha$ limit, unlike the $Y_{\text {apex }}(\alpha)=Y_{m}(\alpha)$ which diverges in this limit. Therefore, the USRSE aspect ratio, $Y_{\text {apex }}(\alpha) / X_{\text {apex }}(\alpha)$ diverges in the small $\alpha$ limit, 


$$
\frac{Y_{\text {apex }}(\alpha)}{X_{\text {apex }}(\alpha)}=\frac{2\left[Y_{m}(\alpha)\right]^{2}}{\pi}=\frac{2}{\pi} \ln \left(\frac{1}{\frac{\pi}{2} \alpha}\right)
$$

Thus, for small $\alpha$ values, USRSEs assume forms that are very elongated along Y-axis. Therefore, the string arclength from the planet to the apex, $s_{\text {apex }}$ is nearly equal to $R_{2, \max }$. Let us calculate $\frac{s_{a p e x}-R_{2, \max }}{\lambda}=S_{\text {apex }}-Y_{\max }$, to the leading order for small- $\alpha$, i.e., large $Y_{m}^{2}$ :

$$
S_{\text {apex }}-Y_{m}=\int_{0}^{Y_{m}} d Y\left[\sqrt{1+\left(\frac{d X}{d Y}\right)^{2}}-1\right]=\int_{0}^{Y_{m}} d Y\left[\frac{1}{\sin (\theta)}-1\right]=-\int_{0}^{\frac{\pi}{2}} d \theta \frac{d Y}{d \theta}\left[\frac{1}{\sin (\theta)}-1\right]
$$

Thus, by Eqs. (98) and (103), to the leading order for small- $\alpha$, i.e., large $Y_{m}$.

$$
\mathrm{S}_{\text {apex }}-\mathrm{Y}_{\mathrm{m}}=\int_{0}^{\theta_{\text {eff }}} \mathrm{d} \theta \frac{\tan (\theta)}{\sqrt{\mathrm{Y}_{\mathrm{m}}^{2}+\ln \left[\cos ^{2}(\theta)\right]}}\left[\frac{1}{\sin (\theta)}-1\right]=\frac{1}{\mathrm{Y}_{\mathrm{m}}} \int_{0}^{\theta_{\text {eff }}} \mathrm{d} \theta \frac{1}{\sqrt{1+\frac{1}{\mathrm{Y}_{\mathrm{m}}^{2}} \ln \left[\cos ^{2}(\theta)\right]}}\left[\frac{1-\sin (\theta)}{\cos (\theta)}\right]
$$

By Eq. (104), for a large $Y_{m}^{2}$ (whence, by Eq. (97), $\theta_{\text {eff }} \rightarrow \pi / 2$ ), one obtains the asymptotic result,

$$
S_{\text {apex }}-Y_{m}=\frac{1}{Y_{m}} \int_{0}^{\pi / 2} d \theta \frac{1-\sin (\theta)}{\cos (\theta)}=\frac{\ln (2)}{Y_{m}(\alpha)}
$$

Thus, for small $\alpha$ (i.e., large $Y_{m}$ ),

$$
S_{\text {apex }}-Y_{m}=\frac{\ln (2)}{Y_{m}(\alpha)}=\frac{\ln (2)}{\sqrt{\ln \left(\frac{1}{\frac{\pi}{2} \alpha}\right)}}
$$

By Eq. (106) and (101), $S_{\text {apex }}-Y_{m}$ and $X_{\text {apex }}$ scale the same way in the large $Y_{m}^{2}$ limit, with universal ratio,

$$
\frac{\mathrm{S}_{\mathrm{apex}}-\mathrm{Y}_{\mathrm{m}}}{\mathrm{X}_{\mathrm{apex}}}=\frac{2 \ln (2)}{\pi}
$$

\section{Copyrights}

Copyright for this article is retained by the author(s), with first publication rights granted to the journal.

This is an open-access article distributed under the terms and conditions of the Creative Commons Attribution license (http://creativecommons.org/licenses/by/4.0/). 